\title{
Better technology for neuronal manipulation
}

\author{
Modelling diseases of the central and peripheral nervous systems and effectively treating neurological disorders via \\ neuronal manipulation requires far better biomaterials and technology than are currently available.
}

U nderstanding how brain tissue changes owing to developmental disorders, trauma or degenerative conditions - such as epilepsy, autism, traumatic brain injury, brain tumours and Parkinson's disease - requires physiological tissue models that can recapitulate the molecular-, cellular- and tissue-level hallmarks of the specific disease. However, the currently primitive understanding of the development of the human brain limits the ability to generate functional brain tissue in vitro. Also, although all neurons share features such as the generation of action potentials, brain tissue consists of a plethora of neuronal and glial subtypes, many still to be defined, each with its own morphology, a unique set of expressed transcription factors and, ultimately, serving a specific function (for example, sensory, motor or relay; or producing a specific neurotransmitter, such as gamma aminobutyric acid, dopamine or serotonin). Moreover, the cellular and neuronal-network organization of the mammalian brain is highly complex, with hundreds of neuronal cell types typically arranged in interconnected clusters or layers.

Still, differentiation protocols allow for the derivation, from pluripotent stem cells, of motor neurons and of some excitatory and inhibitory neurons of the human cortex. Some neuronal subtypes can also be induced by the direct reprogramming of fibroblasts, yet the efficiencies are low and the use of viral vectors for the intracellular delivery of transcription factors hinders the use of the cells for therapeutic or regeneration purposes. Biomaterials that recapitulate microenvironments such as those in the brain can boost the reprogramming efficiency, as shown by Seung-Woo Cho, Hoon-Chul Kang and colleagues in this issue. The researchers show that hydrogels made from decellularized extracellular matrix from human brains increase the efficiency of the direct conversion of mouse fibroblasts into induced neurons with mature morphology and phenotypes, and that the produced neurons improved the locomotion ability of mice that had a stroke induced before the cells were transplanted into the ischaemic region of their brains. The bulk matrices also favoured the differentiation of human stem cells into neurons.

Other natural matrices, such as the widely used Matrigel (a protein gel derived from mouse tumour cells that includes components of brain extracellular matrix), can also provide the physiological environment needed for the generation of human neural tissues from induced neurons differentiated from human embryonic stem cells, as shown by Halil Tekin and co-authors. By using RNA sequencing to study the transcriptomic profiles of tissues made from co-cultures of human neuronal cells and mouse astrocytes, the authors show how the culture conditions (in particular the composition of Matrigel and alginate mixtures and the degree of crosslinking) can be used to modulate the engineered tissues to make them resemble specific brain regions, developmental stages and neurologicaldisease conditions. Also, by using clustered regularly interspaced short palindromic repeats (CRISPR) to knock out three genes implicated in amyotrophic lateral sclerosis, the authors generated engineered tissues that could in principle be used to study the genetics of neurodegenerative diseases.

Although standard CRISPR-mediated genome editing is easy to carry out in vitro, making the technology work in vivo, in particular to edit genes in neurons in the brain so as to treat a neurological disease, is far from straightforward, more so when the editing technique has to be designed to be as safe as possible for eventual use in humans. Hye Young Lee, Niren Murthy and colleagues show that genes in neurons, astrocytes and microglia in mouse brains can be edited by injecting intracranially the ribonucleoproteins Cas9 or Cpf1 and a guide RNA, both encapsulated in gold nanoparticles wrapped in endosomedisruptive polymer (these nanoparticles were first used to edit the mutation that causes Duchenne muscular dystrophy in mice). The researchers show that mice with a form of autism (fragile X syndrome, caused by a single gene) can be rescued from the exaggerated and repetitive behaviour associated with the syndrome by injecting, into the striatum (a brain region that mediates the formation of memory), nanoparticles incorporating a guide RNA targeting a gene for a neurotransmitter receptor. When compared with the use of viruses to insert Cas9 genes into cells in vivo, the use of nanoparticles is safer, allows for better control of the gene editing, and causes fewer off-target effects.

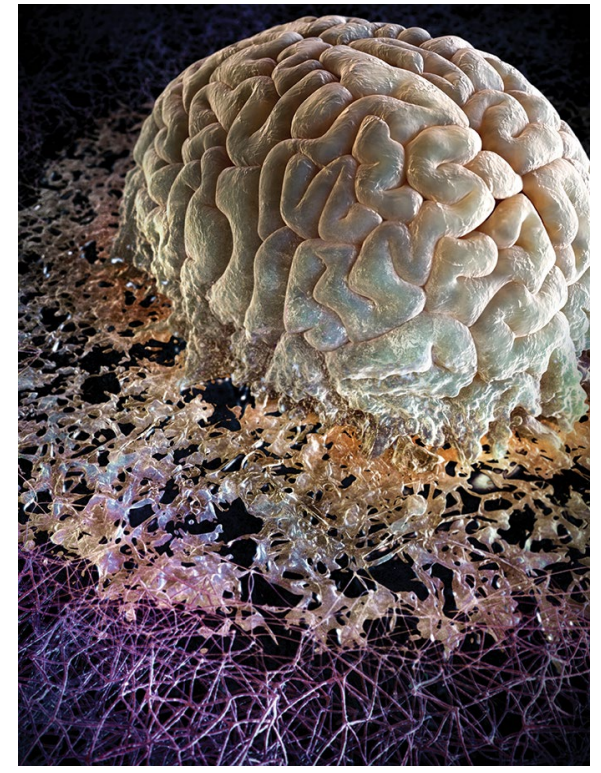

Decellularized brain matrix. Credit: Image by Seung-Woo Cho, Yonsei University and Younghee Lee, 3D CUBE Graphic

Yet most diseases do not have a defined genetic component. To understand neuronal function or to address neuronal disease, it is most useful to alter or control the activity of populations of neurons or nerves. For instance, to stimulate specific peripheral nerves, for example to recover sensation, instigate motor action or block pain signals from reaching the brain, cuff electrodes or intrafascicular electrodes are typically used. Yet invasive electrodes have limitations regarding the sort of sensory or motor fibres that can be selectively stimulated. Optogenetic techniques (which have mainly been applied to stimulate brain regions with light by means of neurons genetically modified to express light-sensitive ion channels) can also be used for stimulating peripheral nerves. Hugh Herr and colleagues demonstrate that two subpopulations of nerve fibres within the sciatic nerve can be stimulated non-invasively to drive opposing muscle movements in the ankles of rats by injecting intramuscularly adeno-associated viruses coding for two newly discovered light-gated ion channels (two opsins, one responding to blue light and the other to red light). The researchers also used the same optogenetics approach to inhibit tremor of 
the muscles downstream of the illumination point on the sciatic nerve. However, the viral transfection of the opsonins caused some muscle atrophy.

In the brain, selective stimulation of neural circuits in specific regions can in principle be achieved via local injections of genes or cells, or by optogenetic, electric or magnetic brain stimulation. Yet to treat chronic brain diseases, implanted stimulation electrodes are currently the only viable option. Mikhail Shapiro and co-authors report a non-invasive approach that combines transient opening of the blood-brain barrier via focused ultrasound, viral gene delivery, and designer receptors that are only activated by designer drugs. The approach allows for the stimulation or inhibition of specific neurons (via genetic means) in specific brain regions (enabled by focused ultrasound) and at desired times (through the administration of the designer drug). The authors demonstrate that the approach could inhibit the formation of contextual fear memories in mice by inhibiting excitatory neurons in the hippocampus.

Instead of using genetic means to modulate the activity of peripheral or central neurons, one can use soft and flexible optoelectronic materials, as shown by Bozhi Tian and collaborators. The researchers demonstrate that interfaces made of silicon in the shape of nanowires, microscopic membranes or flexible macroscopic meshes can be used to modulate, on illumination with light, intracellular calcium dynamics (via the photothermal effect), the release of neurotransmitters from brain slices (via the generation of capacitive currents), and brain activity in anaesthetized mice (via the generation of capacitive and Faradaic currents), respectively. The researchers provide a set of design principles for the fabrication of such silicon biointerfaces, which should facilitate the development of new neuromodulation strategies.
In patients, neuromodulation of the central and peripheral nervous systems is carried out mostly invasively (through implantable electrodes and stimulators, as in deep brain stimulation to treat Parkinson's disease and in spinal-cord stimulation to relieve chronic pain following spinal surgeries). Non-invasive electrical and magnetic methods, such as transcranial direct current stimulation and transcranial magnetic stimulation, only provide therapeutic benefit for a few neurological conditions (such as neuropathic pain). As exemplified by the proof-of-principle biomaterials-based strategies and genetic and non-genetic technologies highlighted here and described in this issue, better technology is needed for modelling and modulating the brain and its peripheral neuronal extensions non-invasively (or minimally invasively), safely and effectively.

Published online: 11 July 2018 https://doi.org/10.1038/s41551-018-0274-2 